\title{
Retração do solo e relação com as propriedades físicas e matéria orgânica de Latossolos e Nitossolos do Sul do Brasil
}

\author{
Soil retraction and relationship with the physical properties and organic matter of \\ Latossolos and Nitossolos of the South of Brazil
}

\section{Luana da Silva*, Jackson Adriano Albuquerque, Diego Bortolini, Mariana Bender}

Universidade do Estado de Santa Catarina, Lages, SC, Brasil. *Autor para correspondência: slv.luana@gmail.com

Submissão: 29/07/2019 / Aceite: 22/06/2020

\begin{abstract}
RESUMO
Estudos recentes apontaram que o principal fator de origem do caráter retrátil, observado em Latossolos e Nitossolos, não é a mineralogia. Com isso formulou-se a hipótese de que a retração do solo em Latossolos e Nitossolos tem origem em fatores estruturais do solo, portanto podem ser investigados indiretamente através das propriedades físicas da estrutura do solo. Sendo assim o objetivo deste trabalho foi relacionar a retração com propriedades físicas e a matéria orgânica do solo, investigando de maneira indireta o processo de retração do solo. Foram selecionados seis perfis coletados nos Estados de Santa Catarina e no Rio Grande do Sul que apresentam caráter retrátil e um Vertissolo Ebânico no Rio Grande do Sul. Foram avaliados: granulometria, limites de consistência, densidade do solo e de partículas, porosidade total, microporos e macroporos, área superficial específica, índice de retração, matéria orgânica do solo e retenção de água no solo. A relação entre as propriedades foi realizada através de análise de componentes principais (ACP). Adicionalmente, para analisar o efeito da estrutura $e$ da matéria orgânica no índice de retração, e amostras foram coletadas nos horizontes $A, A B, B A, B t 1$, Bt2, Bt3 do Nitossolo Bruno de Painel, o qual possui um gradiente natural de decréscimo de matéria orgânica no perfil. Neste, as determinações da curva de retenção e índice de retração foram realizadas com amostras de estrutura preservada e alterada. A ACP indicou o índice plasticidade com maior relação com a retração do solo, indicando que a redução do teor de água cria meniscos entre as partículas assim a tensão superficial aumenta, causando uma curvatura do menisco, na interface entre água e ar aproximando as partículas, causando assim a retração do solo. A matéria orgânica tem efeito direto no processo de retração, no entanto, a intensidade da retração é dependente da estruturação do solo.
\end{abstract}

PALAVRAS-CHAVE: caráter retrátil, estrutura.

\begin{abstract}
Recent studies have shown that the main origin factor of the retractable character observed in Latossolos and Nitossolos is not mineralogy. Thus, it was hypothesized that soil shrinkage in Latossolos and Nitossolos originates from soil structural factors, so they can be investigated indirectly through the physical properties of soil structure. Thus, the objective of this work was to relate the shrinkage with physical properties and the organic matter of the soil, indirectly investigating the process of soil shrinkage. Six profiles collected in the states of Santa Catarina and Rio Grande do Sul were selected, which have a retractable character and one Vertissolo Ebânico in Rio Grande do Sul. The following were evaluated: particle size, consistency limits, soil and particle density, total porosity, micropores. and macropores, specific surface area, shrinkage index, soil organic matter and soil water retention. The relationship between the properties was performed through principal component analysis (PCA). Additionally, to analyze the effect of structure and organic matter on shrinkage index, samples were collected on the $A$, $A B, B A, B t 1, B t 2, B t 3$ horizons of the Nitossolo Bruno of Painel, which has a natural gradient of matter decrease organic in profile. In this, the retention curve and shrinkage index determinations were performed with preserved and altered structure samples. The PCA indicated the plasticity index most closely related to soil shrinkage, indicating that the reduction in water content creates menisci between the particles so the surface tension increases causing a meniscus curvature at the interface between water and air bringing the particles closer together, thus causing soil shrinkage. Organic matter has a direct effect on the shrinkage process; however, the shrinkage intensity is dependent on soil structure.
\end{abstract}

KEYWORDS: retractable character, structure. 


\section{INTRODUÇÃO}

Alguns solos brasileiros apresentam característica de forte contração e expansão, nos quais estão inclusos os que possuem alta quantidade de argilominerais expansivos e os com caráter retrátil. Há algum tempo a característica de forte retração em Latossolos e Nitossolos vem sendo investigada, e foi objeto de discussão na Reunião de Classificação e Correlação de Solos que ocorreu em Santa Catarina no ano de 2008, na qual foi sugerida a inclusão do caráter retrátil no Sistema Brasileiro de Classificação de Solos (SiBCS), como critério para enquadramento dos referidos solos. A retração dos solos ainda é um tema pouco pesquisado e sua gênese não está bem esclarecida. Para os pesquisadores, é intrigante a condição de solos com predomínio de minerais não expansivos possuírem retração a ponto de formar fendas, e os blocos de solo se separarem do perfil.

Sendo ainda, esta condição, de pouco conhecimento entre os pesquisadores, torna-se notável a necessidade de investigação deste processo, visto que, não se sabe até o momento de que forma este solo retrai a sua massa. Sobre estes solos, sabe-se até o momento que, TESTONI (2015) e TESTONI et al. (2017) analisando solos dos estados de Santa Catarina e Rio Grande do Sul, verificou que a composição mineralógica dos horizontes A e B da fração argila total foi similar (entre os perfis de Latossolos e Nitossolos analisados), em geral com picos atribuídos a caulinita, e picos com menor intensidade geralmente indicativos da presença de argilominerais de camada 2:1 ou clorita. Também ocorreram picos de menor intensidade, na maioria das amostras, atribuídos à goethita e/ou hematita e a presença de interestratificados. Portanto este trabalho comprovou que os solos com caráter retrátil não têm predominância de argilominerais expansivos.

Com o resultado do trabalho de TESTONI et al. (2017) não se confirmou a hipótese do caráter retrátil ter origem mineralógica. Com isso formulou-se a hipótese de que a retração do solo em Latossolos e Nitossolos tem origem em fatores estruturais do solo, portanto podem ser investigados indiretamente através das propriedades físicas da estrutura do solo.

O objetivo deste trabalho foi relacionar a retração com propriedades físicas e a matéria orgânica do solo, investigando de maneira indireta o processo de retração do solo.

\section{MATERIAL E MÉTODOS}

\section{Características gerais dos solos}

Para avaliar o comportamento das propriedades físicas e matéria orgânica dos solos com caráter retrátil foram selecionados seis perfis de solos coletados nos estados de Santa Catarina e Rio Grande do Sul que apresentam o caráter retrátil identificado à campo, conforme localização geográfica e classificação informadas abaixo na Tabela 1. Além destes foi coletado um Vertissolo Ebânico, em Santana do Livramento, RS, para comparação, visto que os Vertissolos são solos presentes no Brasil que mais apresentam o mecanismo de retração/expansão devido a sua mineralogia 2:1.

Tabela 1. Descrição e localização de seis perfis de solo com presença de caráter retrátil e um Vertissolo Ebânico com mineralogia predominantemente expansiva.

Table 1. Description and location of six soil profiles with retractile character presence and a Vertissolo Ebânico with predominantly expansive mineralogy.

\begin{tabular}{|c|c|c|c|}
\hline Identificação & Classificação & Local & Localização \\
\hline $\mathrm{NB}_{\mathrm{PAl}}{ }^{*}$ & Nitossolo Bruno Distrófico típico & Painel - SC & $\begin{array}{l}\text { 27053'42" S; } \\
50^{\circ} 07^{\prime} 45^{\prime \prime} \mathrm{W}\end{array}$ \\
\hline $\mathrm{LB}_{\mathrm{VAC}}$ & Latossolo Bruno Distrófico típico & Vacaria - RS & $\begin{array}{l}27^{\circ} 22^{\prime} 35^{\prime \prime} \mathrm{S} ; \\
51^{\circ} 05^{\prime} 27^{\prime \prime} \mathrm{W}\end{array}$ \\
\hline $\mathrm{LV}_{\mathrm{CN}}$ & Latossolo Vermelho Distrófico retrático & Campos Novos - SC & $\begin{array}{l}28^{\circ} 30^{\prime} 47 " \mathrm{~S} ; \\
50^{\circ} 53^{\prime} 37 " \mathrm{~W}\end{array}$ \\
\hline $\mathrm{NB}_{\mathrm{PS}}$ & Nitossolo Bruno Distrófico húmico & Ponte Serrada - SC & $\begin{array}{l}26^{\circ} 51^{\prime} 23^{\prime \prime} \mathrm{S} ; \\
52^{\circ} 02^{\prime} 33^{\prime \prime} \mathrm{W}\end{array}$ \\
\hline $\mathrm{NB}_{\text {CUR }}$ & Nitossolo Bruno Distrófico húmico & Curitibanos - SC & $\begin{array}{l}27^{\circ} 22^{\prime} 12^{\prime \prime} \mathrm{S} ; \\
50^{\circ} 34^{\prime} 46^{\prime \prime} \mathrm{W}\end{array}$ \\
\hline LB $_{\text {VAR }}$ & Latossolo Bruno Distrófico nitossólico & Vargeão - SC & $\begin{array}{l}26^{\circ} 51^{\prime} 13^{\prime \prime} \mathrm{S} ; \\
52^{\circ} 05^{\prime} 56^{\prime \prime} \mathrm{W}\end{array}$ \\
\hline $\mathrm{VE}_{\mathrm{SL}}$ & Vertissolo Ebânico Órtico chernossólico & $\begin{array}{l}\text { Santana do Livramento } \\
\text { - RS }\end{array}$ & $\begin{array}{l}30^{\circ} 42^{\prime} 05^{\prime \prime} \mathrm{S} ; \\
55^{\circ} 49^{\prime} 43^{\prime \prime} \mathrm{W}\end{array}$ \\
\hline
\end{tabular}

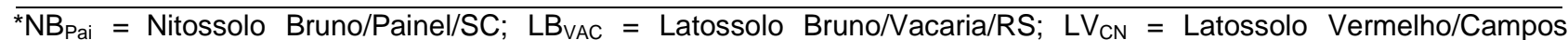
Novos/SC; $\mathrm{NB}_{\mathrm{PS}}=$ Nitossolo Bruno/Ponte Serrada/SC; NB $\mathrm{CUR}=$ Nitossolo Bruno/ Curitibanos/SC; LBVAR: Latossolo Bruno/Vargeão/SC; VE $E_{S L}=$ Vertissolo Ebânico/ Santana do Livramento/RS. 
Todos os solos estudados são originados da formação Serra Geral, que se refere aos derrames e intrusivas que recobrem a Bacia do Paraná, que abrange toda a região centro-sul do Brasil (CPRM 2007). $\mathrm{Na}$ Tabela 2 estão descritas as principais características de formação dos solos estudados. Os solos $\mathrm{NB}_{\text {Pai, }}$, $L V_{C N}, N B_{P S}, N B_{C u r}$ e $L B_{V a r}$ foram obtidas da VIII Reunião Nacional de Correlação e Classificação dos Solos de Santa Catarina (EMBRAPA \& EPAGRI 2008); o LB Vac $_{\text {ac }}$ está descrito em EMBRAPA (2000); e o Vertissolo Ebânico está descrito em REINERT et al. (2007).

Tabela 2. Descrição dos fatores de formação de seis perfis de solo com presença de caráter retrátil e um Vertissolo Ebânico com mineralogia predominantemente expansiva.

Table 2. Description of the formation factors of six soil profiles with a retractile character and an Vertissolo Ebânico with predominantly expansive mineralogy.

\begin{tabular}{|c|c|c|c|c|c|c|c|}
\hline Identificação $^{* * * *}$ & Litologia & Formação & Geológica & Cronologia & Altitude & $\begin{array}{l}\text { Relevo } \\
\text { Local }\end{array}$ & $\begin{array}{l}\text { Vegetação } \\
\text { Primária }\end{array}$ \\
\hline & & Grupo & Formação & & & & \\
\hline $\mathrm{NB}_{\mathrm{PAI}}{ }^{*}$ & Basalto & $\begin{array}{l}\text { São } \\
\text { Bento }\end{array}$ & $\begin{array}{l}\text { Serra } \\
\text { Geral }\end{array}$ & $\begin{array}{l}\text { Jurássico - } \\
\text { Cretáceo }\end{array}$ & $1150 \mathrm{~m}$ & Ondulado & $\begin{array}{l}\text { Floresta } \\
\text { Ombrófila } \\
\text { Mista }\end{array}$ \\
\hline $\mathrm{LB}_{\mathrm{VAC}} \mathrm{C}^{* *}$ & Basalto & $\begin{array}{l}\text { São } \\
\text { Bento }\end{array}$ & $\begin{array}{l}\text { Serra } \\
\text { Geral }\end{array}$ & $\begin{array}{l}\text { Jurássico - } \\
\text { Cretáceo }\end{array}$ & $1000 \mathrm{~m}$ & $\begin{array}{l}\text { Suave } \\
\text { ondulado }\end{array}$ & Campo \\
\hline $\mathrm{LV}_{\mathrm{CN}}^{*}$ & Basalto & $\begin{array}{l}\text { São } \\
\text { Bento }\end{array}$ & $\begin{array}{l}\text { Serra } \\
\text { Geral }\end{array}$ & $\begin{array}{l}\text { Jurássico - } \\
\text { Triássico }\end{array}$ & $939 \mathrm{~m}$ & $\begin{array}{l}\text { Suave } \\
\text { ondulado }\end{array}$ & $\begin{array}{l}\text { Campo } \\
\text { Nativo }\end{array}$ \\
\hline $\mathrm{NB}_{\mathrm{PS}}{ }^{*}$ & Dacito & $\begin{array}{l}\text { São } \\
\text { Bento }\end{array}$ & $\begin{array}{l}\text { Serra } \\
\text { Geral }\end{array}$ & $\begin{array}{l}\text { Jurássico - } \\
\text { Triássico }\end{array}$ & $1065 \mathrm{~m}$ & $\begin{array}{l}\text { Suave } \\
\text { ondulado }\end{array}$ & $\begin{array}{l}\text { Floresta } \\
\text { Ombrófila } \\
\text { Mista }\end{array}$ \\
\hline $\mathrm{NB}_{\text {CUR }}{ }^{*}$ & Basalto & $\begin{array}{l}\text { São } \\
\text { Bento }\end{array}$ & $\begin{array}{l}\text { Serra } \\
\text { Geral }\end{array}$ & $\begin{array}{l}\text { Jurássico - } \\
\text { Cretáceo }\end{array}$ & $1018 \mathrm{~m}$ & $\begin{array}{l}\text { Suave } \\
\text { ondulado }\end{array}$ & $\begin{array}{l}\text { Campo } \\
\text { Nativo }\end{array}$ \\
\hline $\mathrm{LB}_{\mathrm{VAR}}{ }^{*}$ & Dacito & $\begin{array}{l}\text { São } \\
\text { Bento }\end{array}$ & $\begin{array}{l}\text { Serra } \\
\text { Geral }\end{array}$ & $\begin{array}{l}\text { Jurássico - } \\
\text { Cretáceo }\end{array}$ & $1043 \mathrm{~m}$ & $\begin{array}{l}\text { Suave } \\
\text { ondulado }\end{array}$ & $\begin{array}{l}\text { Floresta } \\
\text { Ombrófila } \\
\text { Mista }\end{array}$ \\
\hline$V E_{S L}{ }^{* \star *}$ & Basalto & Escobar & $\begin{array}{l}\text { Serra } \\
\text { Geral }\end{array}$ & $\begin{array}{l}\text { Cretáceo - } \\
\text { Inferior }\end{array}$ & $233 \mathrm{~m}$ & $\begin{array}{l}\text { Plano a } \\
\text { Ondulado }\end{array}$ & $\begin{array}{l}\text { Campo } \\
\text { Nativo }\end{array}$ \\
\hline
\end{tabular}

Fonte: * EMBRAPA \& EPAGRI (2008); ${ }^{* \star}$ EMBRAPA (2000); ${ }^{* \star *}$ REINERT et al. (2007). ${ }^{* \star \star *}$ NB Pai $=$ Nitossolo Bruno/ Painel/SC; $L_{\text {VAC }}=$ Latossolo Bruno/ Vacaria/RS; $L_{\text {CN }}=$ Latossolo Vermelho/ Campos Novos/SC; NBPS = Nitossolo Bruno/ Ponte Serrada/SC; NB $\mathrm{CUR}_{\mathrm{R}}=$ Nitossolo Bruno/ Curitibanos/SC; $\mathrm{LBVAR}_{\mathrm{V}}=$ Latossolo Bruno/ Vargeão/SC; VE $\mathrm{SL}_{\mathrm{S}}=$ Vertissolo Ebânico/ Santana do Livramento/RS.

\section{Metodologias de Avaliação}

Granulometria: foi obtida pelo método da pipeta, descrito por DAY (1965), utilizando $\mathrm{NaOH} 1 \mathrm{~mol} \mathrm{~L}^{-1}$ como dispersante; a areia total foi obtida por peneiramento úmido e o silte por diferença, todos com três repetições por horizonte;

Argila dispersa em água $(A D)$ foi obtida pelo mesmo procedimento, apenas sem o uso do dispersante químico;

Grau de Floculação (GF): obtido através da fórmula: $G F=\frac{A T-A D}{A T}$,

Consistência do solo: O limite de liquidez (LL), limite de plasticidade (LPI), limite de pegajosidade (LPeg) e o índice de pegajosidade (IP) foram determinados pelo Método de Casagrande, conforme os critérios definidos por Atterberg e descritos em EMBRAPA (2017).

Matéria orgânica do solo (MOS): O teor de carbono orgânico do solo (COS) (Tabela 3) foi determinado pela quantificação elementar pelo equipamento MULTI N/C, 2100 (EMBRAPA 2017), e convertido em teor de matéria orgânica do solo (MOS) através da seguinte fórmula:

$M O S=C O S * 1,724$

\section{Avaliação da relação da retração com teor de matéria orgânica e a estrutura}

Para analisar a relação entre o conteúdo da MOS e a estrutura na retração do solo, foi selecionado o Nitossolo Bruno localizado em Painel, SC ( $\left(\mathrm{NB}_{\mathrm{PAI}}\right)$, o qual apresenta naturalmente um decréscimo gradual de carbono orgânico em seus horizontes. Foram coletadas em cada horizonte (A, AB, BA, Bt1, Bt2 e Bt3), amostras com estrutura alterada e estrutura preservada, representando distintas condições de estrutura do solo, as quais foram processadas da seguinte forma:

- Estrutura preservada 
i. Coleta em anéis volumétricos de volume $141,4 \mathrm{~cm}^{3} \mathrm{~cm}^{-3}$

ii. Anéis foram saturados e tensões padronizadas em $60 \mathrm{~cm}$

iii. Anéis secos em estufa com circulação de ar a $105^{\circ} \mathrm{C}$ por 24 horas

iv. Realizado procedimento de análise de retração do solo

- Estrutura alterada

i. Coleta de solo nos horizontes indicados

ii. Amostras tamisadas na peneira de $2 \mathrm{~mm}$ e secas a $105^{\circ} \mathrm{C}$

iii. Anéis de volume $141,4 \mathrm{~cm}^{3} \mathrm{~cm}^{-3}$ preenchidos de acordo com a densidade de cada horizonte

iv. Anéis foram saturados e colocados em mesa de tensão a $60 \mathrm{~cm}$ de coluna de água durante dois dias

v. Anéis secos em estufa com circulação de ar a $105^{\circ} \mathrm{C}$ por 24 horas

vi. Realizado procedimento de análise de retração do solo

Tabela 3. Teor de matéria orgânica e densidade dos horizontes de um Nitossolo Bruno, localizado em Painel, SC.

Table 3. The organic matter contente and density of the horizons of a Nitossolo Bruno, located in SC, Painel.

\begin{tabular}{ccc}
\hline Horizonte & MOS $\left(\mathrm{g} \mathrm{kg}^{-1}\right)$ & $\mathrm{Ds}\left(\mathrm{g} \mathrm{cm}^{-3}\right)$ \\
\hline $\mathrm{A}$ & 80 & 1,17 \\
$\mathrm{BA}$ & 54 & 1,23 \\
$\mathrm{Bt}_{1}$ & 32 & 1,20 \\
$\mathrm{Bt}_{2}$ & 18 & 1,26 \\
$\mathrm{Bt}_{3}$ & 17 & 1,29 \\
\hline
\end{tabular}

Retração do solo: A retração foi realizada através da análise descrita por SILVA et al. (2017) para o cálculo do índice de retração, e adaptou-se o método também no presente estudo para mensurar a porcentagem de volume de solo retraído.

Densidade do solo (Ds), porosidade total (PT), macroporosidade (Macro), microporosidade (Micro), água disponível (AD), capacidade de campo (CC) e ponto de murcha permanente (PMP): As amostras com estrutura preservada foram saturadas e submetidas a sucções de 1,6 e $10 \mathrm{kPa}$ em mesa de areia (REINERT \& REICHERT 2006), e pressão de 1.500 kPa em câmaras de Richards (RICHARDS 1949). Após isso, as amostras foram secas em estufa à $105^{\circ} \mathrm{C}$ para determinar a Ds, Pt, Macro e Micro.

Densidade de partículas (Dp): determinada segundo método descrito em EMBRAPA (2017).

Área superficial específica (ASE): determinada pelo método do Éter Monoetil Etileno Glicol (EGME) (CARTER et al. 1986).

\section{Análise Estatística}

Para verificar a relação entre as propriedades do solo, com foco naquelas que se relacionam com a retração solo, foi realizada a análise de componentes principais foi realizada conforme procedimento PROC PRINCOMP do programa SAS 9.2 (SAS 2009). Utilizou-se o conjunto de dados com as seguintes variáveis de solo, para os solos com presença do caráter retrátil: IR, Dp, areia, silte, argila, argila em água, GF, MO, ASE, LL, LP, LPeg, IP, PT, Ds, Macro e Microporos.

Para a avaliação da relação da retração com teor de matéria orgânica e a estrutura do solo foi realizada teste de regressão linear da retração obtidas nas amostras dos seis horizontes do Nitossolo Bruno localizado em Painel, SC (NB $\left.\mathrm{PAI}_{\mathrm{I}}\right)$, com valores de densidade do solo nas amostras com estrutura preservada e alterada de cada horizontes.

\section{RESULTADOS E DISCUSSÃO}

Atendendo uma das especificações que pré-estabelece o caráter retrátil (SANTOS et al. 2018), todos os solos estudados estão classificados nas classes texturais argiloso e muito argiloso, visualizado no triângulo textural abaixo de LEMOS e SANTOS (1996) (Figura 1).

Observa-se também que todos os solos apresentaram baixo teor de areia. O teor elevado de argila é fortemente influenciado pelo material de origem bem como pelo intenso processo de intemperismo que ocorre nos solos, evidenciando que os solos estudados são altamente intemperizados.

A Ds no horizonte A variou entre 0,80 e $0,96 \mathrm{~g} \mathrm{~cm}^{-3}$, e no horizonte $B$ entre 0,98 e $1,21 \mathrm{~g} \mathrm{~cm}^{-3}$ (Tabela 4) para os solos retráteis. No horizonte $A$ do Vertissolo a Ds foi de $1,10 \mathrm{~g} \mathrm{~cm}^{-3}$. Sabe-se que solos argilosos com Ds inferiores a $1,25 \mathrm{~g} \mathrm{~cm}^{-3}$ dificilmente apresentam restrições ao crescimento e desenvolvimento de raízes (REINERT \& REICHERT 2006). No caso do Vertissolo, mesmo a Ds não sendo 
restritiva ao crescimento de raízes, suas características mineralógicas proporcionam limitações físicas como o fendilhamento durante períodos mais secos, alta plasticidade e pegajosidade e baixa condutividade hidráulica e taxa de infiltração (MOUSTAKAS 2012).

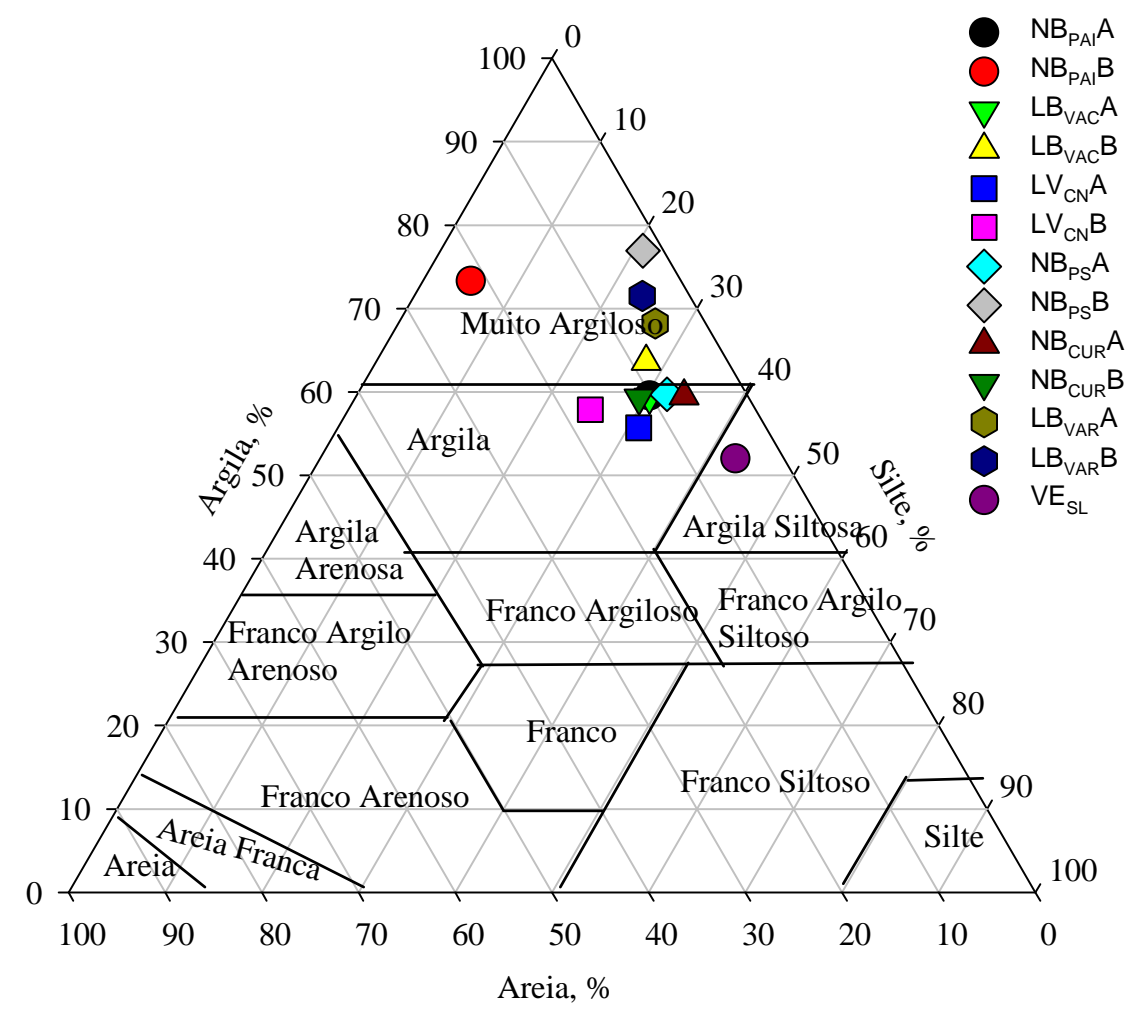

Figura 1. Triângulo textural de seis perfis de solo com presença de caráter retrátil e um Vertissolo Ebânico com mineralogia predominantemente expansíva. Onde: $\mathrm{NB}_{\mathrm{PA}}=$ Nitossolo Bruno/Painel/SC; $\mathrm{LB}_{\mathrm{VAC}}=$ Latossolo Bruno/Vacaria/RS; $\mathrm{LV}_{\mathrm{CN}}=$ Latossolo Vermelho/Campos Novos/SC; $\mathrm{NB}_{\mathrm{PS}}=$ Nitossolo Bruno/Ponte Serrada/SC; $\mathrm{NB}_{\mathrm{CUR}}=$ Nitossolo Bruno/Curitibanos/SC; $\mathrm{LB}_{\mathrm{VAR}}=$ Latossolo Bruno/Vargeão/SC; VE $\mathrm{SL}_{\mathrm{S}}=$ Vertissolo Ebânico/Santana do Livramento/RS.

Figure 1. Textural triangle of six soil profiles with retractile character presence and an Ebony Vertisol with predominantly expansive mineralogy. In which: $N B_{P A l}=$ Nitossolo Bruno/Painel/SC; $L B_{V A C}=L$ atossolo Bruno/Vacaria/RS; $L V_{C N}=$ Latossolo Vermelho/Campos Novos/SC; $N B_{P S}=$ Nitossolo Bruno/Ponte Serrada/SC; $N B_{C U R}=$ Nitossolo Bruno/Curitibanos/SC; $L B_{V A R}=$ Latossolo Bruno/Vargeão/SC; VE $S L=$ Vertissolo Ebânico/Santana do Livramento/RS.

A Dp média dos solos da região geralmente está próxima de $2,65 \mathrm{~g} \mathrm{~cm}^{-3}$, valor este que reflete a densidade média dos argilominerais presentes em cada solo (FERREIRA 2010). Para os solos retráteis no horizonte $A$ à $D p$ variou entre 2,49 a $2,66 \mathrm{~g} \mathrm{~cm}^{-3}$, enquanto no horizonte $B$ entre $2,60 \mathrm{a}_{2,69 \mathrm{~g} \mathrm{~cm}}^{-3}$, enquanto o Vertissolo apresentou $D p$ de $2,22 \mathrm{~g} \mathrm{~cm}^{-3}$. Em geral a $D p$ nos horizontes $A$ foi inferior à do horizonte $\mathrm{B}$, isto ocorre devido a maior quantidade de MOS no $\mathrm{A}$, a qual possui densidade em torno de 1,2 $\mathrm{g}$

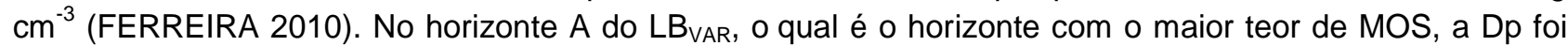
menor, $2,49 \mathrm{~g} \mathrm{~cm}^{-3}$. Essa distinção de valores mostra a variação mineralógica e orgânica desses solos, onde maiores teores de MOS diminuem a Dp, enquanto maior composição por óxidos de Fe eleva a Dp (FERREIRA 2010).

A PT da maioria dos solos e horizontes foi alta, geralmente superando $0,60 \mathrm{~cm}^{3} \mathrm{~cm}^{-3}$ (Tabela 4). Na maioria dos casos, foi maior no horizonte $A$, devido a maior contribuição da MOS e seus efeitos na estruturação do solo. Somente para o horizonte $B$ do $\operatorname{LB}_{V a r}$ a PT foi maior $\left(0,66 \mathrm{~cm}^{3} \mathrm{~cm}^{-3}\right)$ do que no horizonte $A\left(0,61 \mathrm{~cm}^{3} \mathrm{~cm}^{-3}\right)$, o que pode ser atribuído à predominância de estrutura forte, muito pequena, granular (pó-de-café) no horizonte B. Na maioria dos solos a PT é composta principalmente por Micro, que variaram de 0,41 a $0,51 \mathrm{~cm}^{3} \mathrm{~cm}^{-3}$ para os Nitossolos e de 0,37 a $0,52 \mathrm{~cm}^{3} \mathrm{~cm}^{-3}$ para Latossolos. A Macro variou entre 0,10 e $0,30 \mathrm{~cm}^{3} \mathrm{~cm}^{-3}$, sendo geralmente maior no horizonte $A$, devido ao efeito da MOS na formação de estruturas granulares. O Vertissolo possui PT de $0,62 \mathrm{~cm}^{3} \mathrm{~cm}^{-3}$, baixa Macro $\left(0,08 \mathrm{~cm}^{3} \mathrm{~cm}^{-3}\right)$ comparada aos solos retráteis, e Micro elevada $\left(0,55 \mathrm{~cm}^{3} \mathrm{~cm}^{-3}\right)$, valores estes semelhantes aos encontrados por PÉRTILE (2015). 
Tabela 4. Valores médios de densidade solo (Ds), densidade de partículas (Dp), porosidade total (PT), macroporosidade (Macro), microporosidade (Micro), matéria orgânica do solo (MOS), areia, silte, argila, limite de liquidez (LL), limite de plasticidade (LP), limite de pegajosidade (LPeg), índice de plasticidade (IP) e área superficial específica (ASE).

Table 4. Mean values of soil density (Ds), particle density (Dp), total porosity (PT), macroporosity (Macro), microporosity (Micro), soil organic matter (MOS), sand, silt, clay, liquidity limit (LL), plasticity limit $(L P)$, stickiness limit (LPeg), plasticity index (IP) and specific surface area (ASE).

\begin{tabular}{|c|c|c|c|c|c|c|c|c|c|c|c|c|}
\hline Solo & Hor & Ds & Dp & PT & Macro & Micro & MOS & LP & LL & LPeg & IP & ASE \\
\hline & & $----g c$ & $n^{-3}--$ & ------- & $\mathrm{m}^{3} \mathrm{~cm}^{-3}$ & ----- & $--\mathrm{g} \mathrm{kg}^{-1}--$ & 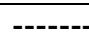 & $---g$ & -1- & --- & $m^{2} g^{-1}$ \\
\hline $\mathrm{NB}_{\mathrm{PAl}}$ & $A$ & 0,91 & 2,64 & 0,68 & 0,16 & 0,52 & 75 & 0,50 & 0,58 & 0,62 & 0,08 & 130 \\
\hline $\mathrm{NB}_{\mathrm{PAl}}$ & B & 1,21 & 2,66 & 0,60 & 0,10 & 0,50 & 47 & 0,45 & 0,51 & 0,56 & 0,07 & 146 \\
\hline LB $_{\text {VAC }}$ & A & 0,94 & 2,60 & 0,70 & 0,18 & 0,52 & 92 & 0,51 & 0,60 & 0,65 & 0,08 & 144 \\
\hline LB $_{\text {VAC }}$ & B & 1,06 & 2,65 & 0,66 & 0,17 & 0,49 & 74 & 0,47 & 0,56 & 0,62 & 0,09 & 166 \\
\hline $\mathrm{LV}_{\mathrm{CN}}$ & A & 0,82 & 2,57 & 0,67 & 0,30 & 0,37 & 65 & 0,45 & 0,53 & 0,63 & 0,08 & 150 \\
\hline $\mathrm{LV}_{\mathrm{CN}}$ & B & 1,02 & 2,60 & 0,63 & 0,16 & 0,47 & 63 & 0,52 & 0,57 & 0,69 & 0,10 & 154 \\
\hline $\mathrm{NB}_{\mathrm{PS}}$ & A & 0,80 & 2,61 & 0,66 & 0,26 & 0,40 & 74 & 0,37 & 0,47 & 0,55 & 0,18 & 90 \\
\hline $\mathrm{NB}_{\mathrm{PS}}$ & B & 0,98 & 2,66 & 0,63 & 0,12 & 0,51 & 44 & 0,41 & 0,60 & 0,61 & 0,19 & 159 \\
\hline $\mathrm{NB}_{\mathrm{CUR}}$ & A & 0,90 & 2,52 & 0,64 & 0,19 & 0,45 & 72 & 0,43 & 0,57 & 0,59 & 0,13 & 90 \\
\hline $\mathrm{NB}_{\text {CUR }}$ & B & 1,07 & 2,60 & 0,61 & 0,17 & 0,44 & 40 & 0,44 & 0,55 & 0,59 & 0,11 & 124 \\
\hline LB VAR $_{\text {VAR }}$ & A & 0,96 & 2,49 & 0,61 & 0,14 & 0,47 & 95 & 0,51 & 0,56 & 0,60 & 0,05 & 119 \\
\hline LB $_{\text {VAR }}$ & B & 1,01 & 2,69 & 0,66 & 0,21 & 0,45 & 26 & 0,42 & 0,51 & 0,54 & 0,09 & 122 \\
\hline$V_{E_{S L}}$ & A & 1,10 & 2,22 & 0,62 & 0,08 & 0,55 & 109 & 0,65 & 0,70 & 0,71 & 0,05 & 245 \\
\hline
\end{tabular}

Para os solos retráteis os teores de MOS foram maiores em todos os horizontes $\mathrm{A}$, sendo que o

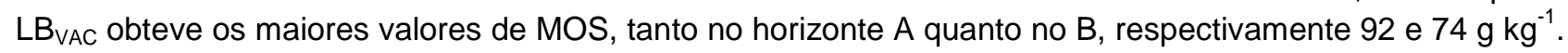
No horizonte $A$ o solo com menor teor de MOS foi o $L V_{C N}$ e no horizonte $B$ o $L_{\text {VAR }}$ (Tabela 4). O Vertissolo possui teor de MOS superior aos solos retráteis, $109 \mathrm{~g} \mathrm{~kg}^{-1}$, isto porque as condições da região do Planalto do Rio Grande do Sul favorecem o acúmulo de MOS devido ao clima mais frio e a posição do solo na paisagem, o qual se encontra no terço inferior da encosta (STRECK et al. 2008).

As propriedades do solo relacionadas a consistência do solo, LL, LP, LPeg e IP, estão apresentadas na Tabela 4. O resultado das forças físicas de coesão e adesão atuando sobre as partículas do solo com diferentes umidades resulta na consistência do solo (HILLEL 1982). A princípio, o conceito de consistência do solo compreende as propriedades que agem de acordo com as forças de adesão e coesão, resistência à compressão e ao esforço cisalhante, friabilidade, plasticidade e pegajosidade, em 1911 foram definidos, pelo cientista sueco A. Atterberg, os denominados limite de liquidez e de plasticidade.

O conhecimento dos limites e dos estados de consistência do solo é importantíssimo nas ações de manejo do solo de acordo com a condição de umidade ideal do solo (KONDO 1998), sendo que a friabilidade é a umidade mais indicada para o preparo do solo e a plasticidade é preocupante em relação a suscetibilidade de compactação (DIAS JUNIOR 1996).

O Vertissolo possui maiores teores de água retida em todos os limites de consistência comparado aos solos retráteis. Para os solos com caráter retrátil, o LP no horizonte $A$ variou de $0,37 \mathrm{~g} \mathrm{~g}^{-1}$ a $0,51 \mathrm{~g} \mathrm{~g}^{-1}$, foi maior nos $L B_{V A C}$ e $L B_{V A R}, 0,51 \mathrm{~g} \mathrm{~g}^{-1}$, no horizonte $B$ o maior valor de $L P$ foram observados no $\operatorname{LB}_{V A C}(0,52$ $\mathrm{g} \mathrm{g}^{-1}$ ) e o menor no NBPS $\left(0,41 \mathrm{~g} \mathrm{~g}^{-1}\right)$. O LL no horizonte $A$, variou de 0,47 a $0,60 \mathrm{~g} \mathrm{~g}^{-1}$, e no horizonte $B$ de 0,51 a $0,60 \mathrm{~g} \mathrm{~g}^{-1}$, variação atribuída ao alto teor de argila e MOS dos mesmos solos. O LPeg representa uma condição de umidade superior ao LP, a partir da qual o solo encontra-se já suficientemente molhado para adquirir propriedade de aderir a outros objetos (BAVER et al. 1972), e no horizonte A dos solos em questão observamos valores entre 0,55 e $0,65 \mathrm{~g} \mathrm{~g}^{-1}$ e no horizonte $\mathrm{B}, 0,54$ e $0,69 \mathrm{~g} \mathrm{~g}^{-1}$. 
O IP para os solos retráteis foi maior no $N_{P S}$ nos horizontes $A$ e $B$, e o menor no horizonte $A$ de $\operatorname{LB}_{\text {VAR }}\left(0,05 \mathrm{~g} \mathrm{~g}^{-1}\right)$, o IP é a diferença entre os limites de liquidez e de plasticidade, expresso em porcentagem e pode ser interpretado, em função da massa de uma amostra, como a quantidade máxima de água que pode lhe ser adicionada, a partir do seu limite de plasticidade, de modo que o solo mantenha a sua consistência plástica, sendo que o Vertissolo possui o mesmo valor IP.

A ASE foi maior no horizonte $B$ do $L_{V A C}$, e menor nos horizontes $A$ do $N_{P S}$ e $N B_{C U R}$. A ASE é afetada principalmente pela distribuição das frações granulométricas, mineralogia e teor de MOS do solo. $O$ Vertissolo possui ASE mais elevada que os solos retráteis principalmente pelo mais elevado teor de MOS, e da mineralogia predominantemente esmectitica (KÄMPF et al. 2012).

Desta forma, observa-se que os solos apresentam variação nos seus atributos como Ds, porosidade, consistência e ASE. Com isso, os processos de retração e expansão do solo também diferiam entre os solos, a Figura 2 apresenta os valores do IR dos horizontes dos solos. O IR do Vertissolo foi maior do que dos demais solos retráteis, característica já esperada devido ao seu alto grau de contração naturalmente observado. Dos solos com caráter retrátil o horizonte $A$ do $N_{P S}$ e $L_{\text {VAC }}$ apresentaram maior IR, e os menores foram para ambos horizontes do $L V_{C N}$. Como é possível perceber o IR independe de ser horizonte A ou B, segundo TESTONI (2015) em todos os solos estudados, a composição mineralógica dos horizontes A e B da fração argila total foi similar, em geral com pontos atribuídos a caulinita, e com menor intensidade picos geralmente indicativos da presença de argilominerais de camada 2:1 ou clorita, e com menor intensidade na maioria das amostras também foram observados picos atribuíveis à goethita e/ou hematita.

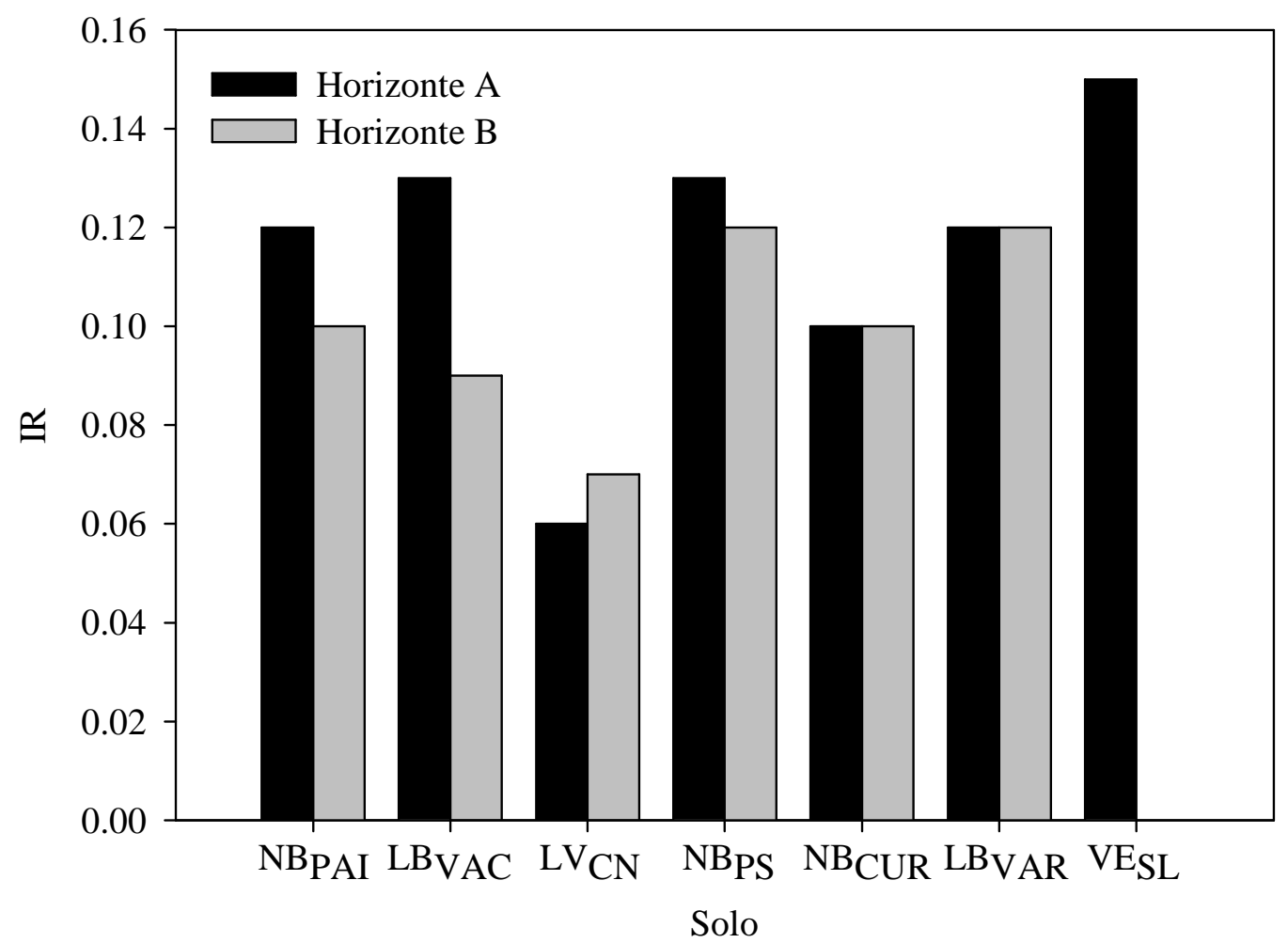

Figura 2. Índice de retração dos solos* determinado através do método do preenchimento do anel com areia - IR. Onde: $\mathrm{NB}_{\mathrm{PAl}}=$ Nitossolo Bruno/Painel/SC; $\mathrm{LB}_{\mathrm{VAC}}=$ Latossolo Bruno/Vacaria/RS; $\mathrm{LV}_{\mathrm{CN}}=$ Latossolo Vermelho/Campos Novos/SC; $\mathrm{NB}_{\mathrm{PS}}=$ Nitossolo Bruno/Ponte Serrada/SC; $\mathrm{NB}_{\mathrm{CUR}}=$ Nitossolo Bruno/Curitibanos/SC; $\mathrm{LB}_{\mathrm{VAR}}=$ Latossolo Bruno/Vargeão/SC; VE $\mathrm{SL}_{\mathrm{S}}=$ Vertissolo Ebânico/ Santana do Livramento/RS.

Figure 2. Soil retraction index ${ }^{*}$ determined by the method of filling the ring with sand - IR. In which: $N B_{P A I}=$ Nitossolo Bruno/Painel/SC; $L B_{V A C}=$ Latossolo Bruno/Vacaria/RS; $L V_{C N}=$ Latossolo Vermelho/Campos Novos/SC; $N B_{P S}=$ Nitossolo Bruno/Ponte Serrada/SC; $N B_{C U R}=$ Nitossolo Bruno/Curitibanos/SC; $L B_{V A R}=$ Latossolo Bruno/Vargeão/SC; $V E_{S L}=$ Vertissolo Ebânico/Santana do Livramento/RS.

Considerando que a mineralogia é similar em todos os horizontes amostrados, deste ponto assumimos que a retração está relacionada a mais propriedades do solo, e estes fazem com que ela seja maior ou menor durante o processo de secamento. Para apontar quais relações são importantes neste processo, foi realizada a análise de componentes principais (ACP) estre todos os atributos analisados 
(Figura 3). A ACP é uma técnica de estatística multivariada que objetiva transformar um conjunto de dados originais em outro conjunto de variáveis numa mesma dimensão (HONGYU 2015), isto quer dizer que, cada componente principal é uma combinação linear de todas as variáveis originais (JOHNSON \& WICHERN 1998). A técnica permite agrupar indivíduos de uma população segundo a variação de suas características (HONGYU 2015). Foram extraídos dois componentes, sendo que o primeiro componente responsável por $33 \%$ da variância dos dados, e o segundo componente responsável por $25 \%$ da variância dos dados.

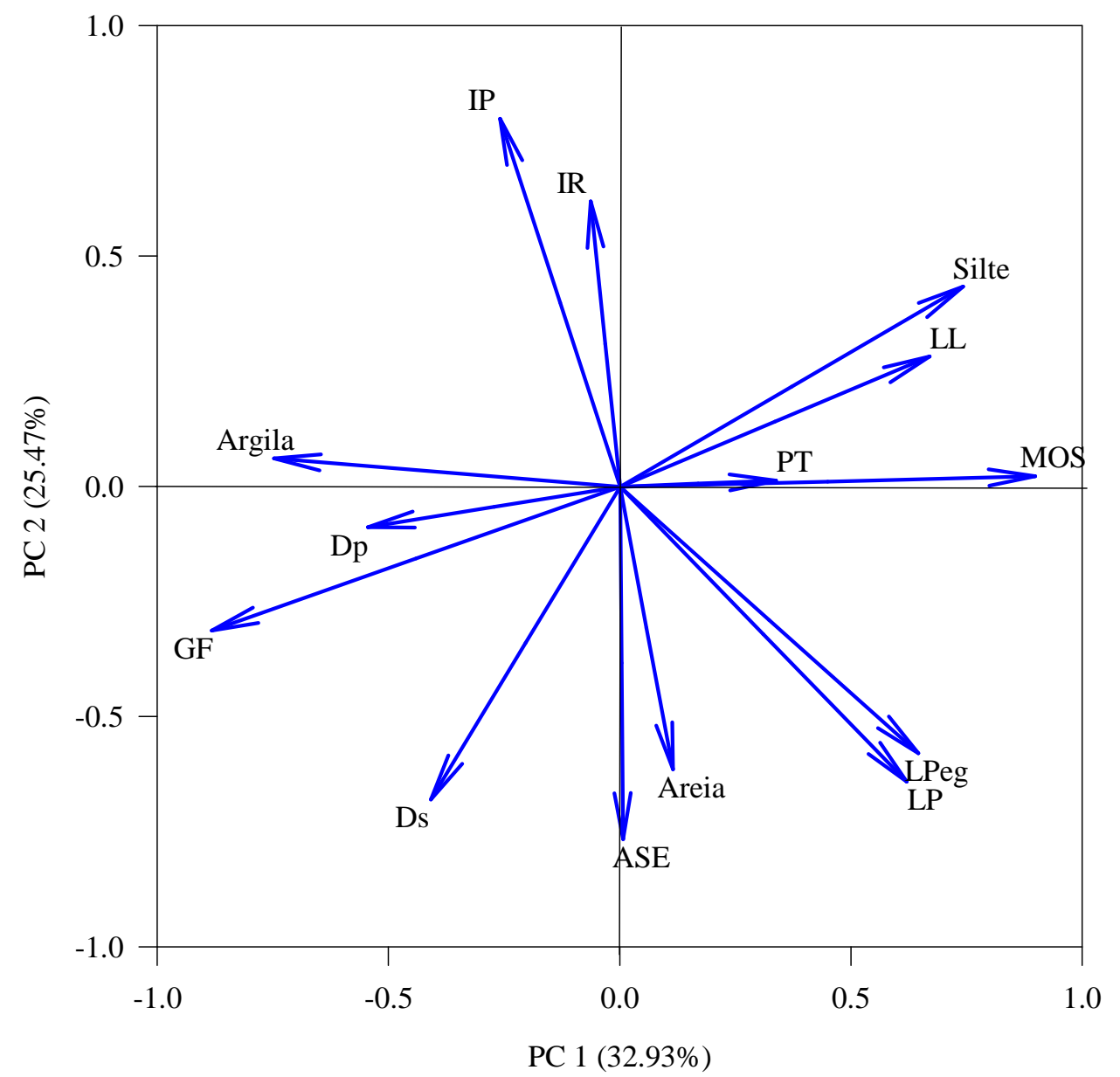

Figura 3. Análise de componentes principais das propriedades físicas do solo. Onde: Argila: argila; Areia: areia; Silte: silte; Ds: densidade do solo; ASE: área superficial do solo; Dp: densidade de partículas; LP: limite de plasticidade; LPeg: limite de pegajosidade; IP: índice de pegajosidade; LL: limite de liquidez; PT: porosidade total; MOS: matéria orgânica do solo; GF: grau de floculação e IR: índice de retração.

Figure 3. Principal components analysis of soil physical properties. In which: Argila: clay; Areia: sand; Silte: lime; Ds: soil density; ASE: soil surface area; Dp: particles density; LP: plasticity limit; LPeg: stickiness limit; IP: stickiness index; LL: liquidity limit; PT: total porosity; MOS: soil organic matter; GF: flocculation degree and IR: retraction index.

Pela ACP o atributo com maior relação com o IR foi o IP. Em geral, segundo NELSON \& MILLER (1992), a plasticidade é um indicador do potencial de expansão, considerando que em solos que apresentam uma ampla variação de umidade possuem um comportamento plástico. Nesta variação de umidade, a água (através da força dos meniscos) afeta a estrutura do solo de maneira a retraí-lo com redução do teor de água no solo. Em resumo, os solos retráteis tem esta característica pois a redução do teor de água cria meniscos entre as partículas (solo não saturado) (KÄMPF \& CURI 2003), e a partir de um determinado potencial matricial a tensão superficial aumenta, causando uma curvatura do menisco, na interface entre água e ar. Com isso, nos potenciais matriciais mais negativos, a força de adesão das moléculas de água com a superfície das partículas e a de coesão entre as moléculas de água aproxima as partículas do solo. Este efeito se intensifica até o ponto do limite de contração, que o solo passa por uma contínua perda de umidade, mas não altera seu volume. Neste ponto a água está fortemente retida pelo mecanismo da adsorção nas superfícies de partículas. Sendo assim, solos com maior IP possuem maior 
potencial de contração e expansão, logo sua maior relação com o IR.

Normalmente, nos solos com alto teor de MOS, quando passam por um processo de secamento, é possível perceber que a massa de solo se altera, devido estes solos possuírem uma PT alta e quando começam a secar, uma grande quantidade de água deixa a massa. Entretanto, não devemos atribuir essa condição ao processo de retração do solo, pois são fatos distintos. Para exemplificar isto, se assume a hipótese de que os solos com caráter retrátil têm sua mudança de volume de acordo com processos que ocorrem no interior das suas microestruturas, e não decorrente do incremento de MOS (Figura 4).

Sendo assim, foi isolado o fator MOS do fator estrutura do solo, e avaliado o comportamento de um solo com caráter retrátil com incremento natural do teor de MOS entre os horizontes do perfil. Para isso utilizou-se solos de cada horizonte com amostra com a estrutura preservada e alterada, como pode ser visto na Figura 4.

Figura 4Analisando as distribuições dos poros destas amostras na mesma condição, observa-se que, as amostras com estrutura preservada obtiveram maior IR comparadas com as de estrutura alterada, em todos os pontos que representam os diferentes teores de MOS. No teor mais baixo de MOS, a amostra com a estrutura alterada teve IR de 0,03 , enquanto com estrutura preservada foi de 0,065 . No maior teor de MOS, o IR da amostra com estrutura alterada foi de 0,07 , e com estrutura alterada foi de 0,11 . Este fato nos remete a hipótese de que, a retração do solo está acontecendo no interior da estrutura.

As amostras com estrutura alterada e preservada apresentam aumento do IR com o aumento do teor de MOS. Nas amostras com estrutura preservada o aumento do IR conforme o teor de MOS, teve coeficiente angular que nas amostras com estrutura preservada, demostrando que a alteração na estrutura provou alteração no IR.

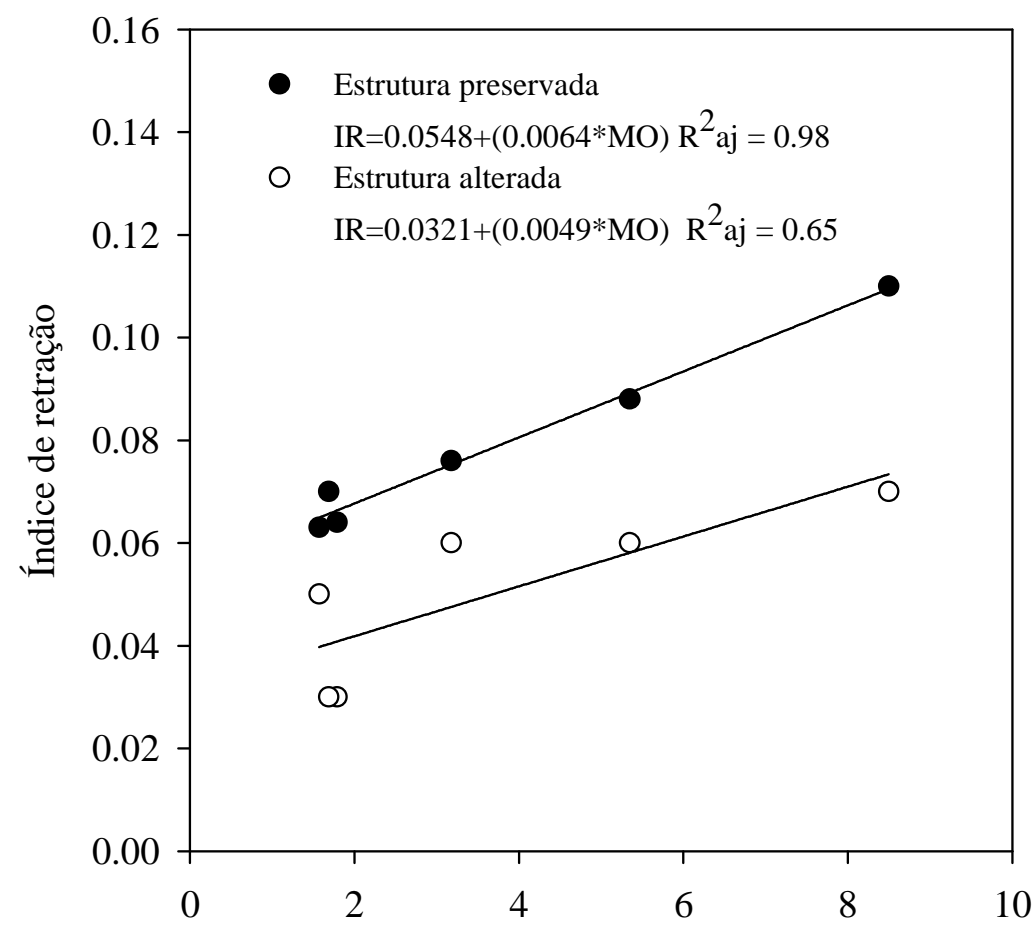

Teor de matéria orgânica, \%

Figura 4. Índice de retração nos diferentes horizontes do Nitossolo Bruno/Painel/SC com diferentes teores de matéria orgânica do solo, em amostras com estrutura preservada e alterada.

Figure 4. Retraction index in the different horizons of the Bruno Nitossolo/Painel/SC with different soil organic matter contents, in samples with preserved and altered structure.

A alteração na estrutura reduziu o volume de poros maiores que $300 \mu \mathrm{m}$ e, principalmente, os menores que $0,2 \mu \mathrm{m}$. Na média de todos os horizontes avaliados, o volume de poros menores que $0,2 \mu \mathrm{m}$ reduziu de $0,45 \mathrm{~cm}^{3} \mathrm{~cm}^{-3}$ nos com estrutura preservada para $0,24 \mathrm{~cm}^{3} \mathrm{~cm}^{-3}$ nos com estrutura alterada. Considerando que: há maior retração nos solos com a estrutura preservada; nesta condição o maior volume de poros são os menores que 0,2 $\mu \mathrm{m}$ (Figura 5); há maior retração ocorre nestes poros; é possível afirmar que a maior retração dos horizontes com estrutura preservada, ocorre quando estes poros perdem água e, com isso, aumentam as forças de adesão e coesão, as quais, portanto, são as responsáveis pela 
modificação na microporosidade do solo.

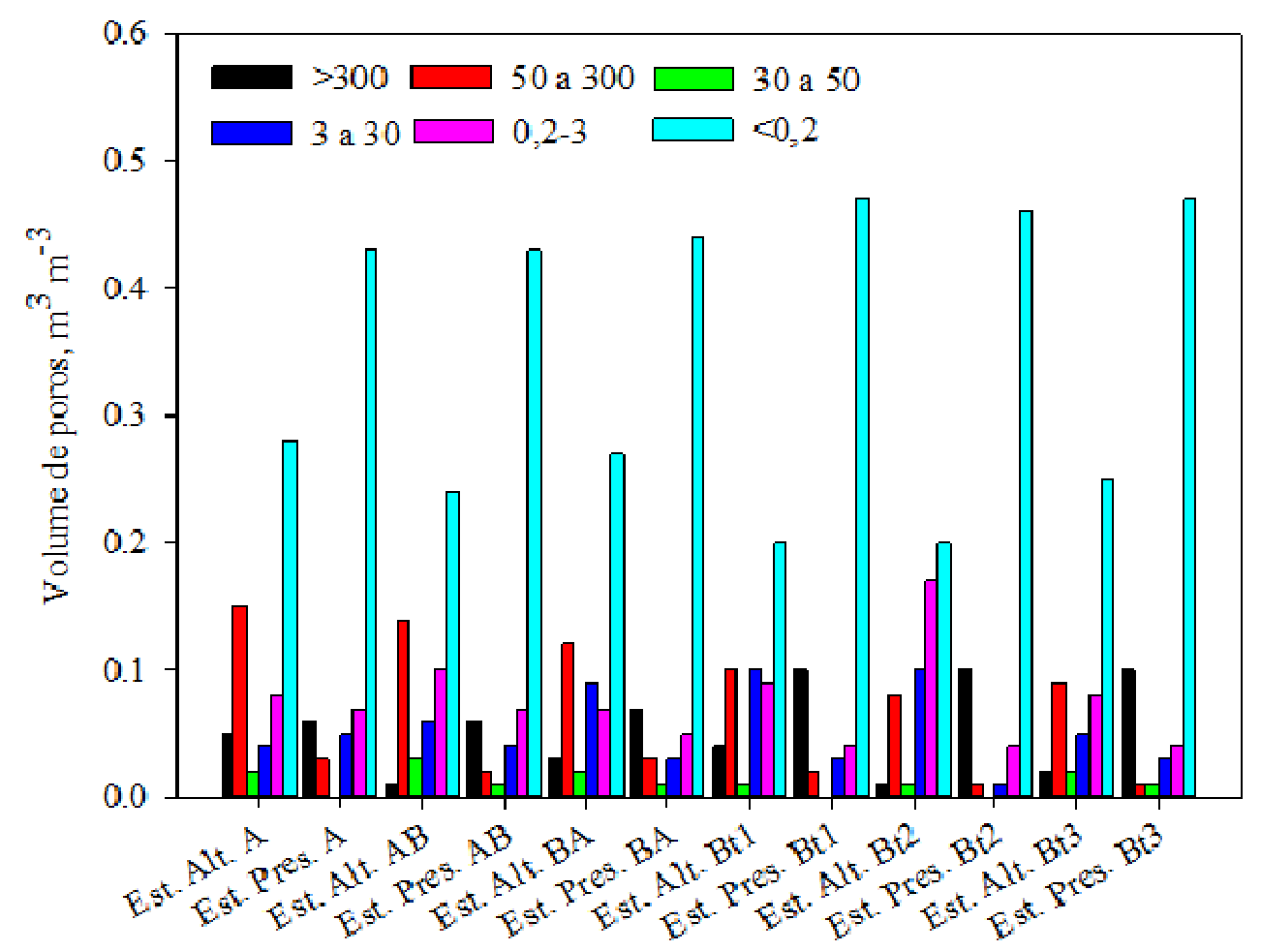

Figura 5. Distribuição de poros dos horizontes A, AB, BA, Bt1, Bt2 e Bt3 do Nitossolo Bruno/Painel/SC, com estrutura preservada e alterada.

Figure 5. Horizontal pores distribution A, AB, BA, Bt1, Bt2 and Bt3 of the Nitossolo Bruno/Painel/SC, with preserved and altered structure.

\section{CONCLUSÃO}

Através dos resultados obtidos foi possível identificar que propriedades físicas do solo como o exemplo do teor de argila e o IP tem relação com retração do solo, sendo possível observar através da ACP. Estas propriedades em particular estão diretamente relacionadas as forças que se modificam com a saída de água do solo (adesão e coesão), onde com a redução do potencial matricial a tensão superficial aumentada, causando uma curvatura do menisco entre água e partículas, na interface entre água e ar aproximando as partículas, causando assim a retração do solo.

A matéria orgânica tem efeito direto no processo de retração, no entanto, a intensidade da retração é dependente da estruturação do solo.

\section{REFERÊNCIAS}

BAVER LD et al. 1972. Soil physics. 4.ed. New York: John Wiley. 498p.

CARTER DL et al. 1986. Specific surface. In: KLUTE A. Methods of soil analysis: Physical and mineralogical methods. 2.ed. Madison: Soil Science Society of America.

CPRM. 2007. Companhia de pesquisa de recursos minerais. Serviço Geológico do Brasil. Programa levantamentos geológicos básicos do Brasil: Agudo (Folha SH.22-V-C-V, escala 1:100.000). Brasília: Ministério de Minas e Energia. $105 p$.

DAY PR. 1965. Particle fractionation and particle-size analysis. In: BLACK CA. (ed.). Methods of soil analysis. Physical and mineralogical methods. 2.ed. Madison: American Society of Agronomy. p.545-567.

EMBRAPA. 2000. Empresa Brasileira de Pesquisa Agropecuária. Guia de excursão de estudos de solos nos estados do Rio Grande do Sul. Colombo: Embrapa Florestas. 222p.

EMBRAPA \& EPAGRI. 2008. VIII RCC Reunião Nacional de Correlação e Classificação de Solos (Santa Catarina). Florianópolis: Epagri/Embrapa. 199p.

EMBRAPA. 2017. Empresa Brasileira de Pesquisa Agropecuária. Manual de métodos de análise de solos. Dados eletrônicos. Rio de Janeiro. Embrapa Solos. 573p.

FERREIRA MM. 2010. Caracterização Física do Solo. In: VAN LIER QJ. (ed) Física do Solo. Viçosa: Sociedade Brasileira de Ciência do Solo. p.2-12.

HILLEL D. 1982. Introduction to soil physics. San Diego: Academic Press. 264p. 
HONGYU K. 2015. Comparação do GGE-biplot ponderado e AMMI-ponderado com outros modelos de interação genótipo x ambiente. Tese (Doutorado em Estatística e Experimentação Agronômica). Piracicaba: USP. 155p.

JOHNSON RA \& WICHERN DW. 1998. Applied multivariate statistical analysis. Madison: Prentice Hall International. $607 p$.

KÄMPF N \& CURI N. 2003. Argilominerais em solos brasileiros. In: CURI N et al (Ed). Tópicos em Ciência do Solo. Viçosa: UFV. p.1-54.

KÄMPF $\mathrm{N}$ et al. 2012. Mineralogia de solos brasileiros. In: KER JC et al. (Eds.). Pedologia: Fundamentos. Viçosa: SBCS. p.207-302.

KONDO MK. 1998. Compressibilidade de três Latossolos sob diferentes usos. Dissertação de Mestrado (Mestrado em Ciência do Solo). Lavras: UFLA. 105p.

LEMOS RC \& SANTOS RD. 1996. Manual de descrição e coleta de solo no campo. 3.ed. Campinas: Sociedade Brasileira de Ciência do Solo. 83p.

MOUSTAKAS NKA. 2012. Study of Vertisol genesis in North Eastern Greece. Catena. 92: 208-215.

PÉRTILE P. 2015. Propriedades mecânicas em micro e mesoescala de solos do Rio Grande do Sul. Tese de Doutorado (Doutorado em Ciência do Solo). Santa Maria: UFSM. 164p.

REINERT DJ \& REICHERT JML. 2006. Coluna de areia para medir a retenção de água no solo: protótipos e teste. Ciência Rural 36: 1931-1935.

REINERT DJ et al. 2007. Principais Solos da Depressão Central e Campanha do Rio Grande do Sul: Guia de Excursão. 2.ed. Santa Maria: UFSM. 47p.

RICHARDS L. 1949. Methods of measuring moisture tension. Soil Science 58: 95-112.

SAS. 2009. Procedures Guide for Personal Computers. 3.ed. Cary: SAS Institute.

SANTOS HG et al. 2018. Sistema Brasileiro de Classificação de Solos. 5.ed. Brasília: Embrapa. 355p.

SILVA L et al. 2017. Methods for Quantifying Shrinkage in Latossolos (Ferralsols) and Nitossolos (Nitisols) in Southern Brazil. Revista Brasileira de Ciência do Solo 41: e0160364.

STRECK EV et al. 2008. Solos do Rio Grande do Sul. Porto Alegre: EMATER/RS-ASCAR. 222p.

TESTONI SA. 2015. Mineralogia da fração argila de Latossolos e Nitossolos Brunos e Vermelhos com caráter retrátil do sul do Brasil. Dissertação de Mestrado (Mestrado em Ciência do Solo). Lages: UDESC. 155p.

TESTONI AS et al. 2017. Clay Mineralogy of Brazilian Oxisols with Shrinkage Properties. Revista Brasileira de Ciência do Solo. 41: e0160487. 